WORKING PAPER · NO. 2021-21

\title{
The Analytic Theory of a Monetary Shock
}

Fernando E. Alvarez and Francesco Lippi

FEBRUARY 2021

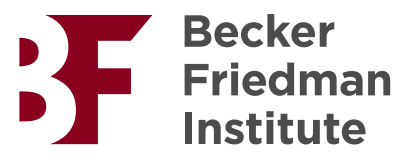




\section{THE ANALYTIC THEORY OF A MONETARY SHOCK}

Fernando E. Alvarez

Francesco Lippi

February 2021

The first draft of this paper is from March 2018, which is 250 years after the birthdate of Jean Baptiste Fourier, from whose terrific 1822 book we respectfully adapted our title. We benefited from conversations with Isaac Baley, Lars Hansen, and Demian Pouzo. We also thank our discussants Sebastian Di Tella and Ben Moll, and participants in "Recent Developments in Macroeconomics" conference (Rome, April 2018), the "Second Global Macroeconomic Workshop" (Marrakech), the 6th Workshop in Macro Banking and Finance in Alghero, and seminar participants at the FBR of Minneapolis and Philadelphia, the European Central Bank, LUISS University, UCL, EIEF, the University Di Tella, Northwestern University, the University of Chicago, the University of Oxford, CREI-UPF, the London School of Economics, Universitat Autonoma Barcelona, Bocconi University, and the 2019 EFG meeting in San Francisco.

(C) 2021 by Fernando E. Alvarez and Francesco Lippi. All rights reserved. Short sections of text, not to exceed two paragraphs, may be quoted without explicit permission provided that full credit, including $\odot$ notice, is given to the source. 
The Analytic Theory of a Monetary Shock

Fernando E. Alvarez and Francesco Lippi

February 2021

JEL No. E5,E50

\section{ABSTRACT}

We propose an analytical method to analyze the propagation of a once-and-for-all shock in a broad class of sticky price models. The method is based on the eigenvalue- eigenfunction representation of the dynamics of the cross-sectional distribution of firms' desired adjustments. A key novelty is that, under assumptions that are appropriate for low-inflation economies, we can approximate the whole profile of the impulse response for any moment of interest in response to an aggregate shock (any displacement of the invariant distribution). We present several applications and discuss extensions.

Fernando E. Alvarez

University of Chicago

Department of Economics

1126 East 59th Street

Chicago, IL 60637

and NBER

f-alvarez1@uchicago.edu

Francesco Lippi

Luiss University

and EIEF

francescolippi@gmail.com

An online appendix is available at http://www.nber.org/data-appendix/w28464 


\section{Introduction}

Economists are often faced with the analysis of dynamic high dimensional objects, such as cross sectional distributions of incomes, assets, markups, and other economic variables of interest. This is the case when studying impulse response functions, namely the dynamics of selected moments of some distribution of interest. We present a powerful method for such analyses that typically require solving the partial differential equation that characterizes the time evolution of the distribution. The method is the eigenvalue-eigenfunction decomposition that allows to solve the partial differential equation through a neat separation of the timedimension from the state-dimension, providing a tractable solution to a non-trivial problem.

Our setup allows us to analytically characterize the whole set of eigenvalues-eigenfunctions and thus to compute the whole profile of the impulse response function, or the whole set of relevant frequencies, for a broad class of class of sticky price models. These models include versions of Taylor (1980), Calvo (1983), Golosov and Lucas (2007), a version of the Calvoplus model by Nakamura and Steinsson (2010), a generalization of the Calvo-plus model to arbitrary random menu costs as in Dotsey, King, and Wolman (1999) and Caballero and Engel (1999, 2007), multi-product models as in Midrigan (2011), Bhattarai and Schoenle (2014) and Alvarez and Lippi (2014), and the model with "price-plans" as in Eichenbaum, Jaimovich, and Rebelo (2011) and Alvarez and Lippi (2019a). In these models firms are hit by idiosyncratic shocks and face a price setting problem featuring (possibly random) menu costs, as well as "price-plans" (i.e. the possibility of choosing two prices instead of a single

one upon resetting). These problems are computationally intensive and numerical solutions may hinder a clear understanding of the propagation mechanism. The approach we propose greatly facilitates the solution of such models, with a simple-to-derive analytic form that unveils the key forces and deep parameters behind the dynamics.

The paper is organized as follows: Section 2 defines the setup of the analysis. Section 3 presents our main result, namely the analytic representation of the impulse response function. Several applications are explored in Section 4, including an application to the sticky-price 
setup developed by Caballero and Engel $(1999,2007)$, where price setting behavior is modelled through generalized-hazard functions that can fit a wide range of theoretical models and empirical price-setting patterns. Most of the analysis assumes an economy with no inflation and symmetric payoffs. Section 5 discusses the robustness with respect to moderate degrees of drift and asymmetry. Avenues for future work, particularly the analysis of models with strategic complementarities, are reviewed in Section 6.

Novelty and relation to the literature. The main novelty of our analysis is the analytic characterization of the whole profile of the impulse response function (IRF). This differs from previous analyses that used the eigenvalue-eigenfunction decomposition to study selected frequencies of interest. For instance, Hansen and Scheinkman (2009) have used the decomposition to model the low frequencies associated to long-run risk in asset pricing, and Gabaix, Lasry, Lions, and Moll (2016) have used it to study the slowest moving frequencies in the dynamics of the distribution of incomes. Besides the focus on a different research question, the main methodological difference compared to these papers is that we characterize all the eigenvalues and eigenfunctions of the dynamical system, while previous papers only characterized the dominant eigenvalue, namely the one that dies at the slowest rate. This is important since we can reproduce the entire IRF, as opposed to obtaining a bound on the long run rate of convergence, which is what the dominant eigenvalue gives. We prove that in our setup the dominant eigenvalue is irrelevant for the IRF of output, since its associated eigenfunction is orthogonal to the function of interest. Even more revealing, we show that using only the dominant eigenvalue as a test of the model dynamics might be misleading: we show that the ranking between the half-life implied by the dominant eigenvalue is not necessarily matched by the ranking of the persistence of the monetary non-neutrality. From a technical stand point, the reason we obtain more information -i.e. all the eigenvalues and eigenfunctions- is that the set of eigenvalues is discrete in our case, while in Gabaix et al. (2016) there is continuum of eigenvalues-this, in turns, is due to the compactness of our operator. 
Our representation of the whole profile of the impulse response function significantly enriches previous analytic results on the impact effect of shocks, such as Caballero and Engel (2007), or analytic results on the cumulated impulse response to shocks such as Alvarez, Le Bihan, and Lippi (2016) and the extension developed by Baley and Blanco (forthcoming) and Alexandrov (2020) for problems with drift and asymmetries. The results also provide straightforward characterizations for several features of interest for a large class of sticky price models, such as duration analysis and the dynamics of any moment of interest after an aggregate shock. Finally, the analysis of the driftless case applies to shocks of any size as well as to shocks to higher moments, such as uncertainty shocks, differently from previous analytic investigations focusing mostly on approximations for small monetary shocks, as in e.g. Gertler and Leahy (2008); Alvarez, Le Bihan, and Lippi (2016); Baley and Blanco (forthcoming).

\section{Set up}

This section introduces the main objects of the analysis. First we introduce a benchmark sticky-price model that is used as the baseline setup and, in Section 4, to illustrate several applications of interest. Second, we set up a standard mathematical definition of the impulse response and establish an equivalence result for symmetric problems.

\subsection{The firm's price setting problem}

This section lays out the price setting problem solved by a firm in the "Generalized Calvoplus" model. In this model the firm is allowed to change prices either by paying a fixed menu cost or upon receiving a random free adjustment opportunity (a menu cost equal to zero). We allow the firm to pay a flow cost $c$ to affect the rate at which this free adjustment opportunities arrive. The setup has elements of the Calvo-plus model, first developed by Nakamura and Steinsson (2010), which nests several models of interest, from the canonical 
menu cost problem to the pure Calvo model. It has also elements of the model developed by Woodford (2009) and Costain and Nakov (2011), where firms can affect the probability of a price change. In Section 4.5 we sketch a model, first proposed by Caballero and Engel (1999) and fully analyzed in Alvarez, Lippi, and Oskolkov (2020), which is equivalent to model presented below but where menu cost are fully random, instead of just having a two point distribution as in the Calvo-plus model.

The firm Ss problem in the Generalized Calvo-plus model. We describe the price setting problem for a firm in steady state. The firm's cost follows a Brownian motion with variance $\sigma^{2}$ and drift $\mu$, where the latter is due to inflation. The price gap $x$ is defined as the price currently charged by the firm relative to the price that will maximize current profits, which is proportional to the firm cost (measured as the log of the ratio between these prices). The firm can change its price at any time by paying a fixed cost $\psi>0$. Additionally, in each time period of length $d t$, if the firm pays a flow cost $c(z) d t$, then it obtains a free adjustment opportunity with with probability $z d t$. The firm policy is summarized by the two barriers $\underline{x}, \bar{x}$, the optimal return point $x^{*}$, and the optimal adjustment rate $z \equiv \xi(x)$ as a function of $x \in(\underline{x}, \bar{x})$. The function $\xi(\cdot)$ is what Caballero and Engel $(1993,1999,2007)$ called a generalized hazard function.

The optimal policy is to change the price when the gap $x$ reaches either of two barriers, $\underline{x}<\bar{x}$, or when the free adjustment opportunity occurs. In either case, at the time of a price change, the firm sets a new price, thus resetting the price gap to $x^{*}$. For this reason we refer to $x^{*}$ as the reinjection point. Price changes are given by $x^{*}-x(\tau)$, where $\tau$ is the stopping time at which either one of the barriers is hit, or a free adjustment opportunity arrives.

The flow cost of the firm is given by $R(x)+c(\xi(x))$, where $R(x)$ is the difference between the static maximum profits and $c(\cdot)$ was defined above. We assume that: $\left(a_{1}\right) R$ and $c$ are convex and non-negative, with $R(0)=0$ and $c(0)=0,\left(a_{2}\right)$ and $c$ is weakly increasing. The firm minimizes expected discounted cost, with a discount rate $r>0$. The value function $v$ 
satisfies:

$$
r v(x)=R(x)+\mu v^{\prime}(x)+\frac{\sigma^{2}}{2} v^{\prime \prime}(x)+\min _{z \geq 0} c(z)+z\left[v\left(x^{*}\right)-v(x)\right] \text { for } x \in[\underline{x}, \bar{x}]
$$

as well as value matching, smooth pasting, optimality of the return point given by $v(\bar{x})=$ $v(\underline{x})=v\left(x^{*}\right)+\psi, v^{\prime}(\underline{x})=v^{\prime}(\bar{x})=v^{\prime}\left(x^{*}\right)=0$, and the first order condition for $z$ which yields the optimal policy function $\xi(x)$ that solves $c^{\prime}(\xi(x))=v(x)-v\left(x^{*}\right)$ for all $x \in(\underline{x}, \bar{x})$.

The setup nests the Calvo-plus model of Nakamura and Steinsson (2010). If $c(z)=0$ for $0 \leq z \leq \zeta$ and $c(z)=\bar{c}>0$ for $z \geq \zeta$, and if $\bar{c}$ is large enough, then the optimal policy is $\xi(x)=\zeta$, which gives rise to the Calvo-plus model, namely one where a constant poisson arrival rate of free adjustment opportunities coexists with the possibility of deliberate price adjustments at a cost $\psi$.

Our results will also apply to the case where the state space is unbounded. Notice that if $\psi=+\infty$ then we have $\bar{x}=-\underline{x}=\infty$, i.e. an unbounded state space. However, for this case we will require an extra condition, namely that $\left(a_{3}\right)$ if $\psi=+\infty$ then $\lim _{z \rightarrow \infty} c^{\prime}(z)=+\infty$ and that $\lim _{x \rightarrow \pm \infty} R(x)=+\infty$. An immediate implication of these assumptions is that $\lim _{x \rightarrow \pm \infty} \xi(x) \rightarrow \infty$, a condition that will be necessary for Theorem 1 to hold. Intuitively, the fact that the hazard function $\xi$ diverges for large $x$ implies that the probability of adjustment converges to one, mimicking what happens when there is a barrier. Notice that such a condition is violated by a model where the hazard function is constant, such as the Calvo model, while it is satisfied by models where the hazard is quadratic.

Invariant distribution. The density of the invariant distribution for price gaps generated by the policy $\left\{\underline{x}, x^{*}, \bar{x}, \xi(\cdot)\right\}$ solves the Kolmogorov forward equation:

$$
\xi(x) \bar{p}(x)=-\mu \bar{p}^{\prime}(x)+\frac{\sigma^{2}}{2} \bar{p}^{\prime \prime}(x) \text { for } x \in[\underline{x}, \bar{x}], x \neq x^{*}
$$

with boundary conditions at the exit points, unit mass, i.e. $\bar{p}(\underline{x})=\bar{p}(\bar{x})=0, \int_{\underline{x}}^{\bar{x}} \bar{p}(x) d x=1$, and $\bar{p}$ continuous at all $x$. We will use $\bar{P}(x)$ to denote the CDF of this density. 


\subsection{The impulse response}

The mathematical set up for the impulse response analysis is made of the following objects: the law of motion of the Markov process $\{x(t)\}$ for each individual firm, the function of interest $f(x)$, the cross-sectional initial distribution of $x$, denoted by $P(x ; 0)$. For instance, using the model defined before to analyze the output impulse response the function of interest is $f(x)=-x$, since each firm's output is inversely proportional to its price gap. Likewise, the initial condition might be given by a small uniform shift of the invariant distribution computed in equation (2), for instance $P(x ; 0)=\bar{P}(x+\delta)$. Several examples will be given in Section 4, which include an initial distribution $P(x ; 0)=\bar{P}(x+\bar{x})$ that is used to study the effect of a large shock $\delta=\bar{x}$ in Section 4.1, the function $f(x)=1$ which we use to study the survival function in Corollary 3, and the quadratic $f(x)=x^{2}$ which is used in Section 4.3 to discuss the response of the dispersion of price gaps following a marginal shock.

At this general level the set-up and definition of an impulse response is closely related to the one in Borovicka, Hansen, and Scheinkman (2014). The law of motion for the process $f(x)$, with $x \in X \equiv[\underline{x}, \bar{x}]$, is also Markov and is described using

$$
\mathcal{H}(f)(x, t)=\mathbb{E}[f(x(t)) \mid x(0)=x]
$$

where the operator $\mathcal{H}$ computes the $t$ period ahead expected value of the function $f: X \rightarrow \mathbb{R}$ conditional on the state $x=x(0)$. Next we describe the initial distribution of $x$, which

we denote by $P(\cdot ; 0): X \rightarrow \mathbb{R}$. This represents the measure of firms that start with value smaller or equal than $x$ at time $t=0$, each of them following the stochastic process described in $\mathcal{H}(f)$, with independent realizations. We allow the initial distribution $P(x ; 0)$ to have countably many mass points. In particular $P$ has a piecewise continuous derivative (density) which we extend to the entire domain, so that $p(\cdot, 0): X \rightarrow \mathbb{R}$, where $P$ can have $K$ jump discontinuities (mass points), denoting the difference between the right and left limits by 
$p_{m}(\cdot ; 0):\left\{x_{k}\right\}_{k=1}^{K} \rightarrow \mathbb{R}$, so that $x_{k}$ is the location of the mass points. ${ }^{1}$ Given the diffusion nature of $x$ such mass points of course will immediately disperse (i.e. turn into a density) one instant after the shock.

We are interested in the standard impulse response function $H$ defined for each $t>0$ as:

$$
H(t ; f, P-\bar{P})=\int_{\underline{x}}^{\bar{x}} \mathcal{H}(f)(x, t)[d P(x ; 0)-d \bar{P}(x)]
$$

where $\bar{P}$ is the invariant distribution of $x$. The impulse response $H$ at time $t$ is the expected value of $f$ computed on the distribution $P(\cdot, t)$ in deviation from its steady state value, where each $x(t)$ has followed the Markov process associated with $\mathcal{H}(\cdot)$ and whose cross sectional distribution at time zero is given by $P(\cdot ; 0) .^{2}$ In other words, for ergodic processes we are forcing the impulse response to go to zero as $t$ diverges. Since we evaluate $H$ only for the difference between two measures, i.e. only for signed measures, we introduce the convenient notation $\hat{P} \equiv P-\bar{P}$ and likewise for the densities $\hat{p}=p-\bar{p}$. Thus

$$
\hat{P}(x) \equiv P(x, 0)-\bar{P}(x) \text { for all } x \in[\underline{x}, \bar{x}]
$$

so that $\hat{P}$ defines the "initial condition" for the impulse response.

We define another impulse response function that uses a stopping time $\tau$, and a modified expectation operator $\mathcal{G}$, defined as:

$$
\mathcal{G}(f)(x, t)=\mathbb{E}\left[1_{\{t \leq \tau\}} f(x(t)) \mid x(0)=x\right]
$$

The operator $\mathcal{G}$ computes the $t$ period ahead expected value of the function $f: X \rightarrow \mathbb{R}$

\footnotetext{
${ }^{1}$ Given our assumption on $P$ we can write the expectations of any function $\nu(x)$ at time zero as:

$$
\int_{\underline{x}}^{\bar{x}} \nu(x) d P(x ; 0)=\int_{\underline{x}}^{\bar{x}} \nu(x) p(x ; 0) d x+\sum_{k=1}^{K} \nu\left(x_{k}\right) p_{m}\left(x_{k} ; 0\right) .
$$

${ }^{2}$ The ergodicity of $\{x\}$ implies that we can write $H(t ; f, P-\bar{P})=\int_{\underline{x}}^{\bar{x}} \mathcal{H}(f)(x, t) d P(x ; 0)-\int_{\underline{x}}^{\bar{x}} f(x) d \bar{P}(x)$.
} 
starting from the value of the state $x=x(0)$, conditional on $x$ surviving. The indicator function $1_{\{t \leq \tau\}}$ becomes zero when the first adjustment following the shock occurs at the stopping time $\tau$.

In the context of the price setting models with $S s$ rules we refer to the operator $\mathcal{H}$ as the one for the problem with "reinjection", i.e. one in which the operator follows a firm forever, i.e. does not stop keeping track of the firm after the first adjustment occurs (at time $\tau$ ). In contrast, we refer to the operator $\mathcal{G}$ as one for the problem without "reinjection", i.e. tracking the firm until the first adjustment. For example, in the sticky price models discussed above the stopping time $\tau$ is given by the occurrence of a price adjustment.

We define the impulse response function $G$ for each $t>0$ as:

$$
G(t ; f, \hat{P})=\int_{\underline{x}}^{\bar{x}} \mathcal{G}(f)(x, t)[d P(x ; 0)-d \bar{P}(x)]
$$

The interpretation of $G(t)$ is the expected value of the cross-sectional distribution of $f$, conditional on surviving, where each $x(t)$ follows the Markov process, and as before the cross-sectional distribution at time zero is given by $P(\cdot ; 0)$ so that the initial condition is $\hat{P} \equiv P(\cdot ; 0)-\bar{P}$.

While $H$ is the impulse response as commonly defined, it turns out that $G$ is simpler to characterize. In Proposition 1 we will establish conditions under which the impulse response $G(t ; f, \hat{P})$ coincides with $H(t ; f, \hat{P})$ for all $t$.

An equivalent, and perhaps simpler, representation of the impulse response function $G(t)$ is obtained by using the transition function $Q_{t}(y \mid x)=\operatorname{Pr}\{x(t)<y, t<\tau \mid x(0)=x\}$, with density function $q_{t}(y \mid x)=\partial_{y} Q_{t}(y \mid x)$. The impulse response function is

$$
G(t ; f, \hat{P})=\int_{\underline{x}}^{\bar{x}} \int_{\underline{x}}^{\bar{x}} f(y) q_{t}(y \mid x) d y d \hat{P}(x)
$$

Our analytical characterization of $G(t)$, which will be given in Theorem 1, can be easily understood by using a finite-dimensional version of equation (8), as we will discuss after 
Corollary 1. In general our interest is to compute $H(t ; f, \hat{P})$ by using the simpler operator $G(t ; f, \hat{P})$ which takes as an argument the signed measure $\hat{P}$.

The initial condition. Our setup encodes the impulse in the initial condition, $\hat{P}(x) \equiv$ $P(x, 0)-\bar{P}(x)$, which denotes the distribution of the state variable $x \in(\underline{x}, \bar{x})$ at time zero in deviation from the invariant distribution. In particular $P(\cdot ; 0)$ describes the cross-sectional distribution of the state immediately after the shock. As time elapses the initial distribution will converge to the invariant distribution $\bar{P}(x)$, tracing out the impulse response for the function of interest $f(x)$. We mentioned above that our method allows the initial distribution to have mass points. This can be useful, for instance, if the initial shock is large enough to displace a non-negligible mass of agents onto the return point $x^{*}$, to compute the survival function of price changes, or to compute the conditional distribution of the state $q_{t}$. Also notice that our formulation allows us to handle a variety of shocks. Several papers have focussed on a small uniform displacement $\delta>0$ of the whole distribution relative to the invariant $\bar{P}(x)$, what Borovicka, Hansen, and Scheinkman (2014) label the "marginal response function", a case that has been studied in the literature, as discussed in the introduction, which we formalize next. We stress, however, that our method can handle any type of initial condition, such as one triggered by a large shock (see Section 4.1), or the one triggered by a higher-order shock (see Appendix C).

Marginal impulse response function. Starting from the invariant distribution $\bar{p}(x)$ and considering a small uniform displacement $\delta>0$ of the whole distribution so that

$$
\hat{p}(x)=\bar{p}(x+\delta)-\bar{p}(x)=\bar{p}^{\prime}(x) \delta+o(\delta)
$$

we have the following definition 
DEFINITION 1. Let the marginal impulse response function to a monetary shock be:

$$
\left.Y(t ; f) \equiv \frac{\partial}{\partial \delta} H(t ; f, \bar{p}(x+\delta)-\bar{p}(x))\right|_{\delta=0} \text { for all } t \geq 0
$$

The marginal impulse response function is the first order expansion of $H(t ; f, \bar{p}(x+\delta)-\bar{p}(x))$ with respect to $\delta$. For future reference notice that using equation (9) we will often write $H\left(t ; f, \bar{p}^{\prime}(x) \delta\right)$ instead of $H(t ; f, \hat{p}(x))$. Since $H(t ; f, \mathbf{0})=0$, where $\mathbf{0}$ denotes the zero function, thus the expansion gives: $H(t ; f, \bar{p}(x+\delta)-\bar{p}(x))=Y(t ; f) \delta+o(\delta)$ where the marginal impulse response $Y(t)$ is measured per unit of the monetary shock.

\subsection{Equivalence of IRF's $H$ and $G$ for symmetric $s S$ problems}

Next we present a proposition for symmetric problems establishing conditions for the standard IRF $H(t)$ to coincide with $G(t)$. We start by defining the notion of a symmetric sS problem. Informally, this amounts to assuming that the firm problem has zero drift and that the firm's return function is symmetric. We then show that for symmetric $s S$ problems $H(t)=G(t)$ for all horizons $t \geq 0$. Let us begin by defining a symmetric problem:

Definition 2. We define a problem to be symmetric if: $\left(s_{1}\right)$ the unregulated state has zero drift, i.e. $\mu=0,\left(s_{2}\right)$ the optimal return point $x^{*}$ is equidistant from the barriers, i.e. $(\bar{x}+\underline{x}) / 2=x^{*}$, and $\left(s_{3}\right) \xi(x)$ is symmetric in $x$ around $x^{*}$.

Let $\{x(t)\}$ be the regulated state, $u\left(x ; t, x^{*}\right)$ be the density of distribution of $x(t)=x$, conditional on $x(0)=x^{*}$. If the decision rules are symmetric, then the density satisfies $u\left(z+x^{*} ; t, x^{*}\right)=u\left(-z+x^{*} ; t, x^{*}\right)$ for all $z \geq 0$. The symmetry of $u\left(\cdot ; t, x^{*}\right)$ follows from the combination of the symmetry of the distribution of a BM without drift, the symmetry of the boundaries relative to $x^{*}$, and the symmetry of $\xi(\cdot)$.

Moreover, if $R$ is symmetric in $x$ relative to $x^{*}$ and if $\mu=0$, then the optimal decision 
rule for the firm's problem are symmetric, as in Definition 2. This follows from using a guess and verify strategy in equation (1) and the boundary conditions, assuming that the value function $v$ is symmetric in $x$ around $x^{*}$.

Proposition 1. Assume the firm's problem is symmetric as in Definition 2. Then if either

(i) the function of interest $f:[\underline{x}, \bar{x}] \rightarrow \mathbb{R}$ is anti-symmetric (and $\hat{P}(\cdot)$ is arbitrary) or

(ii) the initial condition $\hat{P}:[\underline{x}, \bar{x}] \rightarrow \mathbb{R}$, including its mass points, is anti-symmetric (and $f(\cdot)$ is arbitrary)

we have that $G(t ; f, \hat{P})=H(t ; f, \hat{P})$ for all $t$.

See Appendix A for the proof. The proposition's requirement that either the function of interest $f$, or the initial condition $\hat{P}$, is anti-symmetric is not that restrictive for our applications. The main function of interest for the paper, used to compute the IRF for output, is given by $f(x)=-x$ in a large class of models. Also, our benchmark case in this class of models is that the signed measure $\hat{P}$ is anti-symmetric when we consider a small monetary shock.

\section{Analytic Impulse Response Functions}

This section characterizes the impulse response without reinjections given in equation (7), using an analytic solution for the operator $\mathcal{G}(f)(x, t)$ defined in equation $(6)$. The solution allows us to consider models with drift and with asymmetric return point and fully characterize the response to a once and for all shock absent reinjections. As made clear by Proposition 1, the focus on the case without reinjections is without loss of generality for a symmetric model. Next we will present our main result following the logical steps to construct the analytic impulse response function, as opposed to highlighting the elements of the proof that establish 
the result. This choice is inspired by the hope to encourage applications of the methods. See the proof for the mathematics behind the result.

The main analytical tool is the use of eigenvalues and eigenfunctions, for which it is useful to define the inner product:

$$
\langle f, g\rangle \equiv \int_{\underline{x}}^{\bar{x}} f(x) g(x) w(x) d x \quad \text { where } \quad w(x) \equiv e^{\frac{2 \mu}{\sigma^{2}} x}
$$

which we use to define the set of squared integrable functions, $L_{w}^{2}$, for which $\|f\|^{2} \equiv\langle f, f\rangle<$ $\infty$. Note that the "weight" function $w(x)$ is constant in the case of zero drift, i.e $w(x)=1$ if $\mu=0$. Now we introduce the sequence of eigenvalues and eigenfunctions $\left\{\lambda_{j}, \varphi_{j}\right\}_{j=1}^{\infty}$ corresponding to the dynamics of the price gaps characterized by $\left\{\underline{x}, \bar{x}, \xi(\cdot) \mu, \sigma^{2}\right\}$. These eigenfunctions of the problem with drift are obtained by using the functions $\gamma_{j} \in L^{2}$, twice differentiable in $x$ which solves:

$$
\begin{aligned}
& \lambda_{j} \gamma_{j}(x)=\gamma_{j}^{\prime \prime}(x) \frac{\sigma^{2}}{2}-\left(\xi(x)+\frac{1}{2} \frac{\mu^{2}}{\sigma^{2}}\right) \gamma_{j}(x), \text { for all } x \in[\underline{x}, \bar{x}], \\
& \text { s.t. boundary conditions } \gamma_{j}(\bar{x})=\gamma_{j}(\underline{x})=0 \text {, and where } \varphi_{j}(x) \equiv \gamma_{j}(x) e^{-\frac{\mu}{\sigma^{2}} x} .
\end{aligned}
$$

Notice that, given the problem fundamental parameters and policy rules in $\left\{\underline{x}, \bar{x}, \xi(\cdot) \mu, \sigma^{2}\right\}$, finding the eigenvalues and eigenvectors amounts to solving a simple second order differential equation with known boundary conditions. ${ }^{3}$

We summarize well known results for the eigenvalues and eigenfunctions corresponding to the process of the price gap until the next price change defined by $\left\{\underline{x}, x^{*}, \bar{x}, \xi(\cdot), \mu, \sigma^{2}\right\}$, which can be found in Berezinn and Shubin (1991) and Zettl (2010). We assume: $\left[A_{1}\right]$ positive volatility, i.e. $\sigma^{2}>0 ;\left[A_{2}\right]$ non-negative, continuous, generalized hazard function $\xi(\cdot)$; and $\left[A_{3}\right]$ either finite domain, i.e. $-\infty<\underline{x}<\bar{x}<\infty$ or infinite domain $\bar{x}=-\underline{x}=\infty$ with diverging generalized hazard function, i.e. $\xi(x) \rightarrow \infty$ as $x \rightarrow \pm \infty$.

\footnotetext{
${ }^{3}$ Due to the focus on a problem without reinjections the optimal return point $x^{*}$ is not needed to solve for eigenfunctions and eigenvalues.
} 
Proposition 2. Assume that $\left\{\underline{x}, \bar{x}, \xi(\cdot), \mu, \sigma^{2}\right\}$ satisfies assumptions $\left[A_{1}\right],\left[A_{2}\right]$, and $\left[A_{3}\right]$. Then: $\left(E_{1}\right)$ there exists countably many eigenvalues-eigenfunctions pairs solving equation (12); $\left(E_{2}\right)$ the eigenvalues are real, negative, non-repeated, and diverge, i.e. $0>\lambda_{1}>$ $\lambda_{2}>\ldots$, with $\lambda_{j} \rightarrow-\infty$ as $j \rightarrow \infty ;\left(E_{3}\right)$ the eigenfunctions $\left\{\varphi_{j}\right\}$ form an orthonormal base of $L_{w}^{2}$, i.e. $\left\langle\varphi_{i}, \varphi_{j}\right\rangle=\delta_{i j}$, i.e. equals one if $j=i$ and zero otherwise, and any function $f \in L_{w}^{2}$ can be obtained by the projection into the eigenfunctions: $\left\|f-\sum_{j=1}^{\infty}\left\langle f, \varphi_{j}\right\rangle \varphi_{j}\right\|=0$; and $\left[E_{4}\right]$ the eigenfunctions are indexed by their number of interior zeroes, with $\varphi_{j}$ having $j-1$ zeroes on $(\underline{x}, \bar{x})$. If in addition $\left\{\underline{x}, x^{*}, \bar{x}, \xi(\cdot)\right\}$ is symmetric in the sense of Definition 2 , then: $\left[E_{5}\right]$ the eigenfunctions $\varphi_{j}$ indexed by $j=1,3, \ldots$ are symmetric and those indexed by $j=2,4 \ldots$ are antisymmetric.

A special case of interest occurs when the generalized hazard function is constant, i.e $\xi(x)=\zeta$, so that we have the Calvo-plus model. In this case equation (12) takes the form of the well known heat equation studied by Fourier, whose eigenvalues and eigenfunctions are:

$\lambda_{j}=-\left[\zeta+\frac{1}{2} \frac{\mu^{2}}{\sigma^{2}}+\frac{\sigma^{2}}{2}\left(\frac{j \pi}{\bar{x}-\underline{x}}\right)^{2}\right]$ and $\varphi_{j}(x)=\frac{e^{-\frac{\mu}{\sigma^{2}} x}}{\sqrt{(\bar{x}-\underline{x}) / 2}} \sin \left(\frac{[x-\underline{x}]}{[\bar{x}-\underline{x}]} j \pi\right)$ for $j=1,2,3, \ldots$

We are now ready to state our main result:

Theorem 1. Assume that the price gaps are governed by $\left\{\underline{x}, \bar{x}, \xi(\cdot), \mu, \sigma^{2}\right\}$ which satisfies assumptions $\left[A_{1}\right],\left[A_{2}\right]$, and $\left[A_{3}\right]$. Let $f \in L_{w}^{2}$ be piece-wise differentiable, with countably many discontinuities. Furthermore let $\hat{P} \in L_{w}^{2}$ be a piecewise continuous density with at most countably many mass points, $K$. Then the impulse response in equation (7) is:

$$
G(t ; f, \hat{P})=\sum_{j=1}^{\infty} e^{\lambda_{j} t}\left\langle\varphi_{j}, f\right\rangle\left\langle\varphi_{j}, \hat{P} / w\right\rangle+\sum_{j=1}^{\infty} \sum_{k=1}^{K} e^{\lambda_{j} t}\left\langle\varphi_{j}, f\right\rangle \varphi_{j}\left(x_{k}\right) p_{m}\left(x_{k}\right)
$$

where $\langle\cdot, \cdot\rangle$ is the inner product defined in equation (11), each of the eigenvalue-eigenfunction pair satisfies equation (12), and the double summation accounts for $K$ mass points.

Theorem 1 provides a tractable analytic representation of the impulse response function. 
One interesting property of the solution is that it separates the effect of the function of interest $f$, encoded in the projection coefficient $\left\langle\varphi_{j}, f\right\rangle$, from the effect of the initial impulse $\hat{P}$, encoded in the projection coefficient $\left\langle\varphi_{j}, \hat{P} / w\right\rangle$, and from the effect of time, encoded in the eigenvalues $\lambda_{j}$. This implies, for instance, that to analyze shocks of different size one only needs to change the projection coefficients of the initial condition $\hat{P}$, leaving all other coefficients unchanged. Notice that although the eigenvalues (encoding the time decay) are unchanged, the modified coefficients will affect the shape of the impulse response function by changing the "weights" with which the different eigenvalues enter the expression. In Section 4.1 we illustrate an application that compares the propagation of a small vs a large monetary shock in the model of Golosov and Lucas (2007).

In the case where the initial condition $\hat{P}$ has no mass points, the expression consists only of the first part: $G(t ; f, \hat{P})=\sum_{j=1}^{\infty} e^{\lambda_{j} t}\left\langle\varphi_{j}, f\right\rangle\left\langle\varphi_{j}, \hat{P} / w\right\rangle$. Note moreover that, in the case of $\mu \neq 0$, one of the two projections, i.e. either the one for $f$ or the one for $\hat{P}$, is weighted by the function $w(x)=e^{\frac{2 \mu}{\sigma^{2}} x}$, while the other one is not. Where convenient, equation (14) can equivalently be rewritten as

$$
G(t ; f, \hat{P})=\sum_{j=1}^{\infty} e^{\lambda_{j} t}\left\langle\varphi_{j}, f / w\right\rangle\left\langle\varphi_{j}, \hat{P}\right\rangle+\sum_{j=1}^{\infty} \sum_{k=1}^{K} e^{\lambda_{j} t}\left\langle\varphi_{j}, f / w\right\rangle \varphi_{j}\left(x_{k}\right) p_{m}\left(x_{k}\right) w\left(x_{k}\right)
$$

In the case with no drift, i.e. when $\mu=0$, then $w(x)=1$, the two formulations coincide since the projections do not include any weight.

In most of our applications we will use the Calvo-plus model and variations of it, for which we have the eigenvalues and eigenfunctions shown in equation (13). The alert reader will note that in general the eigenfunctions and eigenvalues of equation (12) are, after change in units, the ones of the one dimensional Schrödinger equation with a potential given by $\xi(\cdot)$, and where the "particle" is confined to a box $[\underline{x}, \bar{x}]$. Given the central role that such eigenvalues and eigenfunctions play in quantum mechanics, the solution for this equation are either known in closed form or perturbation techniques have been developed to obtain 
analytical approximations, as summarized in Fernandez (2001). In Section 4.5 we illustrate an application where the generalized hazard function is quadratic, i.e. $\xi(x)=\kappa x^{2}$, which turns out to be one of the most studied quantum mechanical system, namely the quantum harmonic oscillator, for which eigenvalues and eigenfunctions are known in closed form.

Theorem 1 can be readily understood by considering the discrete-time and discrete-state of the representation in equation (8). In this case $q_{t}(y \mid x)$ corresponds to the $\{y, x\}$ element of the $t$-th power of the 1-period transition matrix. Diagonalizing this matrix delivers the eigenvalue and eigenvectors that can be used to compute projections that are equivalent to the ones in equation (14). A straightforward application of Theorem 1 using a limiting argument for the function $f(z ; y, \epsilon)=\mathbf{1}_{\{z \in(y-\epsilon, y+\epsilon)\}} /(2 \epsilon)$, the definition of a density function $q_{t}(y \mid x)$, and an initial condition with all the probability in a mass point at $x$, i.e. $p_{m}(x)=1$, gives a closed form expression for the transition function $q_{t}(y \mid x)$ defined in equation (8):

Corollary 1. Let $(x, y) \in[\underline{x}, \bar{x}]^{2}$. The transition density from $x$ to $y$ in $t$ periods is

$$
q_{t}(y \mid x)=\sum_{j=1}^{\infty} e^{\lambda_{j} t} w(y) \varphi_{j}(y) \varphi_{j}(x)
$$

As was the case for the impulse response function, the theorem delivers a neat separation between the time and the state which gives a simple characterization of the time evolution of the whole distribution after a shock.

\section{Applications}

This section uses Theorem 1 to analyze several economic applications. First an analytic summary of monetary shocks in the standard menu cost model, as in e.g. Golosov and Lucas (2007), for both small and large shocks. We show that this model has a single parameter

determining its behavior, pinned down by the frequency of price changes. Second we use 
Nakamura and Steinsson (2010) Calvo-plus model, encompassing a large class of models that span Golosov-Lucas and Calvo, to discuss the "selection effect", a key mechanisms that explains why different sticky price models yield different real effects (Section 4.2). We show that the selection effect creates a wedge between the duration of price spells and the duration of the aggregate output response. The two durations coincide when there is no selection. Analytically, such a wedge is visible in the magnitude of the eigenvalues that control, respectively, the dynamics of the survival function of prices and the dynamics of aggregate output. Third we present a surprising result proving that, following a marginal monetary shock, the response of even centered moments is zero at all horizons (Section 4.3). This applies for instance to the cross-sectional price dispersion, to the kurtosis of the price changes, as well as to the survival function. Since the cross-sectional price dispersion is the main measure of inefficiency in models with price stickiness, the result implies that in these models such a cost should be measured in an "average" sense, and not as a consequence of a particular shock. Fourth we study the propagation of monetary shocks in models with priceplans introduced by Eichenbaum, Jaimovich, and Rebelo (2011) to model the phenomenon of temporary price changes (prices that move from a reference value for a short period of time and then return to it). These models can be given a full analytical characterization and are particularly interesting because they may yield non-monotone impulse response functions (Section 4.4). Fifth we apply Theorem 1 to a setup pioneered by Caballero and Engel (1993), that uses a "generalized hazard function" to model price setting behavior. This setup allows for a vast variety of empirical price setting patterns to be considered. We analytically solve the model by Caballero and Engel (2007) using a quadratic generalized hazard function as an example (Section 4.5). We conclude by mentioning other tractable applications, including the effect of volatility shocks on the propagation of monetary impulses and models with multi-product firms. 


\subsection{The canonical menu cost model of Golosov and Lucas (2007).}

We solve the canonical menu-cost model, obtained by setting $\mu=0$ and $\zeta=0$ in the problem of Section 2.1, which yields the symmetric inaction region $\underline{x}=-\bar{x}$ with optimal return $x^{*}=0$. To compute the impulse response of output we use $f(x)=-x$ since the contribution of a firm to the deviation of output (relative to steady state) is inversely proportional to its price gap. As prescribed by equation (11), we compute the projection coefficient $\left\langle f, \varphi_{j}\right\rangle$ in equation (14) integrating $f(x)$ against the eigenfunctions $\varphi_{j}(x)$ given in equation (13). This gives

$$
\left\langle f, \varphi_{j}\right\rangle=\frac{4 \bar{x}^{3 / 2}}{j \pi} \text { for } j=2,4,6, \ldots \text {, and }\left\langle f, \varphi_{j}\right\rangle=0 \text { otherwise. }
$$

We first consider a marginal monetary shock so that $\hat{p}(x)=\delta \bar{p}^{\prime}(x)$, as in equation (9). The invariant distribution for this model is readily derived from equation (2) and the associated boundary conditions, which gives the triangular density $\bar{p}(x)=1 / \bar{x}-|x| / \bar{x}^{2}$ for $x \in(-\bar{x}, \bar{x})$. It is apparent that $\bar{p}^{\prime}(x)$ is a step function, equal to $1 / \bar{x}^{2}$ for $x \in[-\bar{x}, 0)$ and equal to $-1 / \bar{x}^{2}$ for $x \in(0, \bar{x}]$. As before we construct the projection coefficients $\left\langle\bar{p}^{\prime}, \varphi_{j}\right\rangle$ integrating $\bar{p}^{\prime}(x)$ against $\varphi_{j}(x)$, this gives

$$
\left\langle\bar{p}^{\prime}, \varphi_{j}\right\rangle=\frac{8}{j \pi \bar{x}^{3 / 2}} \text { if } j=2+4 i \text { for } i=0,1,2, \ldots, \text { and }\left\langle\bar{p}^{\prime}, \varphi_{j}\right\rangle=0 \text { otherwise. }
$$

Thus the impulse response coefficients for equation (14) are:

$$
\left\langle\bar{p}^{\prime}, \varphi_{j}\right\rangle\left\langle f, \varphi_{j}\right\rangle=\frac{32}{(j \pi)^{2}} \text { if } j=2+4 i \text { for } i=0,1,2, \ldots, \text { and }\left\langle\bar{p}^{\prime}, \varphi_{j}\right\rangle\left\langle f, \varphi_{j}\right\rangle=0 \text { otherwise. }
$$

and the (marginal) impulse response of output as defined in equation (10) is:

$$
Y(t)=\sum_{i=0}^{\infty} \frac{32}{((2+4 i) \pi)^{2}} e^{-N \frac{((2+4 i) \pi)^{2}}{8} t}
$$

There are two important properties of the model unveiled by equation (18). First, the model 
dynamics are fully encoded in a single parameter, namely the average number of price changes per period $N=\sigma^{2} / \bar{x}^{2}$. Second, not all eigenvalues matter for output dynamics. In particular, the dominant eigenvalue $\lambda_{1}$, the one related to the very low frequencies, is irrelevant. This is a general property of several models that we further discuss in Corollary 4. As a summary measure, consider the Cumulative IRF or CIR, defined to be $\mathcal{M}(f, \hat{P})=\int_{0}^{\infty} G(t ; f, \hat{P}) d t$. For a marginal shock direct computation using the expression for $Y$ gives $\mathcal{M}(f, \hat{P})=\frac{\delta}{N} \frac{1}{6}$, which agrees with the general formula for CIR in Alvarez, Le Bihan, and Lippi (2016) using Kurtosis. Notice that including only the term for the first eigenvalue with non-zero projection, i.e. $\lambda_{2}$, gives an excellent approximation of the shape of the IRF and of the CIR. Indeed the ratio of the CIR using all the eigenvalues and the CIR using only the second is equal to $96 / \pi^{4} \approx 0.985{ }^{4}$

A large monetary shock. Consider now a large shock that displaces half of the mass of firms outside of the inaction region, namely $\delta=\bar{x} .^{5}$ The cross sectional density right after the shock is $p(x, 0)=-x / \bar{x}^{2}$ for $x \in(-\bar{x}, 0)$, and $p(x, 0)=0$ for $x \in(0, \bar{x})$. Using equation (11) gives the projection coefficients $\left\langle p_{0}, \varphi_{j}\right\rangle=\frac{2}{j \pi \bar{x}^{1 / 2}}$ if $j=2+2 i$ for $i=0,1,2, \ldots$. The odd-indexed projection coefficients are irrelevant since they will be multiplied by the zero coefficients of $f$, see equation (16). We can then normalize by the shock size $\bar{x}$ and use equation (14) to compute the impulse response function (per unit of shock):

$$
Y_{\text {bigshock }}(t)=\sum_{i=0}^{\infty} \frac{8}{((2+2 i) \pi)^{2}} e^{-N \frac{((2+2 i) \pi))^{2}}{8} t}
$$

Notice that this expression depends only on a single parameter, as was the case for the marginal impulse response. Also note that this impulse response features some even-index eigenvalues that were not present in the marginal one (e.g. $j=4,6,8, .$.$) . This implies a$ change of the coefficients, that yields a much smaller persistence of the shocks. This can be easily seen by inspecting the coefficients of the two expressions. We can also compute the

\footnotetext{
${ }^{4}$ See Figure 3 in the working paper version Alvarez and Lippi (2019b).

${ }^{5}$ We are thankful to an anonymous referee for suggesting this application.
} 
CIR for the large shock $\delta=\bar{x}$. In this case we obtain $\mathcal{M}(f, \hat{P})=\frac{\bar{x}}{N} \frac{2}{45}$. We can see that, per unit of shock, the CIR of a large shock is much smaller than the one for a marginal shock, about one fourth in magnitude. Notice that the leading eigenvalue $\lambda_{2}=-N \frac{\pi^{2}}{2}$, that is the same in both impulse responses, is associated with a coefficient that is exactly four times larger in the case of a marginal shock, so that the IRF (per unit of shock) is approximately four times larger at each time $t$.

\subsection{The "selection effect" in the Calvo-plus models}

This section applies Theorem 1 to illustrate why different sticky-price models display different degrees of "selection". The notion of selection, coined by Golosov and Lucas, refers to the fact that firms that adjust prices following a monetary shock are selected from a particular set, such as those that need to make a large adjustment. This contrasts with models where adjusting firms are not systematically selected, such as models where the times of price adjustment are exogenously given such as the Taylor or the Calvo model. It is known that different amounts of selection critically affect the propagation of monetary shocks. We illustrate this result analytically using the Calvo-plus model, a model that nests several special cases featuring different degrees of selection, from Golosov-Lucas to the pure Calvo model.

Decision rules and steady state in the Calvo-plus model. Next we use the decision problem defined in Section 2.1, assume zero inflation $(\mu=0)$ and a quadratic profit function $R(x)=x^{2}$. It is straightforward that $\bar{x}=-\underline{x}>0$ and that the optimal return is $x^{*}=$ 0. Given the policy parameters $\{-\bar{x}, \bar{x}\}$ and the law of motion of the state $d x=\sigma d W$ it is immediate that the eigenvalues-eigenfunctions of the problem are those computed in equation (13). Since the eigenvalues depend on the speed at which prices are changed, we find it convenient to rewrite them in terms of the average number of price changes per unit of time. To this end we compute the expected number of adjustments per unit of time, the

reciprocal of the expected time until an adjustment, $N=\frac{\zeta}{1-\operatorname{sech}(\sqrt{2 \phi})}$ where $\phi \equiv \frac{\zeta \bar{x}^{2}}{\sigma^{2}}$. Note 
that as $\bar{x} \rightarrow \infty$ then $N \rightarrow \zeta$, which is the Calvo model where all adjustment occur after an exogenous poisson shock. As $\zeta \rightarrow 0$ then $N \rightarrow \sigma^{2} / \bar{x}^{2}$ so that the model is Golosov and Lucas. This single parameter $\phi \in(0, \infty)$ controls the degree to which the model varies between Golosov-Lucas and Calvo. Note that with this parameterization we can distinguish between $N$ and the importance of the randomness in the menu cost $\zeta$ vs the width of the barriers, $\bar{x}^{2} / \sigma^{2}$. Indeed $\zeta / N$, the share of adjustment due to random free-adjustments, depends only on $\phi$. We let $\frac{\zeta}{N}=\ell(\phi)$ where this function is defined as $\ell(\phi)=1-\operatorname{sech}(\sqrt{2 \phi})$. The function $\ell(\cdot)$ is increasing in $\phi$, and ranges from 0 to 1 as $\phi$ goes from 0 to $\infty$. The invariant density function $\bar{p}$ solves the Kolmogorov forward $\zeta \bar{p}(x)=\sigma^{2} / 2 \bar{p}^{\prime \prime}(x)$ in the support, except at $x=0$, integrates to one and it is zero at $\pm \bar{x}$. This gives:

$$
\bar{p}(x)=\frac{\theta\left[e^{\theta(2 \bar{x}-|x|)}-e^{\theta|x|}\right]}{2\left(1-e^{\theta \bar{x}}\right)^{2}} \text { for } x \in[-\bar{x}, \bar{x}] \text { where } \quad \theta \equiv \sqrt{2 \zeta / \sigma^{2}}
$$

Finally, using the formula for $N$ and equation (13) we have the eigenvalues:

$$
\lambda_{j}=-\zeta-\frac{\sigma^{2}}{\bar{x}^{2}} \frac{(j \pi)^{2}}{8}=-\zeta\left[1+\frac{(j \pi)^{2}}{8 \phi}\right]=-N \ell(\phi)\left[1+\frac{(j \pi)^{2}}{8 \phi}\right] \text { where } \phi \equiv \frac{\zeta \bar{x}^{2}}{\sigma^{2}}
$$

As before we consider a marginal monetary shock so that $\hat{p}(x)=\delta \bar{p}^{\prime}(x)$, as in equation (9). As above, we express the output IRF $Y(t)$ per unit of the shock $\delta$ as in equation (10). Application of Theorem 1 gives the following:

Corollary 2. The coefficients for the marginal impulse response in the Calvo-plus model are:

$$
\left\langle\bar{p}^{\prime}, \varphi_{j}\right\rangle\left\langle f, \varphi_{j}\right\rangle= \begin{cases}0 & \text { if } j \text { is odd } \\ -2\left[\frac{1+\cosh (\sqrt{2 \phi})}{1-\cosh (\sqrt{2 \phi})}\right]\left[\frac{1}{1+\frac{j^{2} \pi^{2}}{8 \phi}}\right] & \text { if } j \text { is even and } \frac{j}{2} \text { is odd } \\ -2\left[\frac{1}{1+\frac{j^{2} \pi^{2}}{8 \phi}}\right] & \text { if } j \text { is even and } \frac{j}{2} \text { is even }\end{cases}
$$


Interpretation of the Dominant Eigenvalue. The dominant eigenvalue has the interpretation of the asymptotic hazard rate of price changes. In particular, let $h(t)$ be the hazard rate of price spells as a function of the duration of the price spell $t$. Let $\tau$ be the stopping time for prices, i.e. $\tau$ is the first time at which $\sigma W(t)$, which started at $W(0)=0$, either hits $\bar{x}$ or $\underline{x}=-\bar{x}$, or that the Poisson process changes. Let $S(t)$ be the survival function, i.e.: $S(t)=\operatorname{Pr}\{\tau \geq t\}$. Notice that the function of interest to compute the survival function is the indicator $f(x)=1$ for all $t<\tau$. The hazard rate is defined as $h(t)=-S^{\prime}(t) / S(t)$. Application of Theorem 1 gives the following:

Corollary 3. The Survival function $S(t)$ depends only on the odd-indexed eigenvalueseigenfunctions, i.e. $\left(\lambda_{i}, \varphi_{i}\right)$ for $i=1,3,5, \ldots$ Let $h(t)$ be the hazard rate of price changes. Then the dominant eigenvalue $\lambda_{1}$ is equal to the asymptotic hazard rate, i.e.

$$
S(t)=\sum_{j=1,3,5, \ldots} e^{\lambda_{j} t}\left\langle 1, \varphi_{j}\right\rangle \varphi_{j}(0) \quad \text { and } \quad-\lambda_{1}=\lim _{t \rightarrow \infty} h(t)
$$

where we use equation (14) assuming a degenerate random variable concentrated at $x=0$ so that $p_{m}(0)=1$.

Irrelevance of dominant eigenvalue for output IRF. Next we show that the dominant eigenvalue $\lambda_{1}$, as well as all other odd-indexed eigenvalue-eigenfunction pairs, play no role in the output impulse response. Consider the output coefficients in the impulse response, given by equation (16). It is apparent that the coefficients $\left\langle f, \varphi_{j}\right\rangle$ for all the odd-indexed eigenvalues-eigenfunctions $(j=1,3, \ldots)$ are zero, i.e. the loadings of these terms are zero. This implies that the coefficient corresponding to the dominant eigenvalue $\lambda_{1}$ is zero. The first non-zero term, which we call the "leading" eigenvalue, involves $\lambda_{2}$. This is because $\varphi_{j}(\cdot)$ is symmetric around $x=0$ for $j$ odd, and anti-symmetric for $j$ even. Thus: $\int_{\underline{x}}^{\bar{x}} \varphi_{j}(x) f(x) d x=$ $0 \Longrightarrow\left\langle f, \varphi_{j}\right\rangle=0$ for $j=1,3, \ldots$ This happens since all the odd-indexed eigenfunctions $\varphi_{j}$ $(j=1,3, \ldots)$ are symmetric functions, and thus the projection onto them of an asymmetric 
function, such as $f(x)=-x$, yields a zero $\left\langle f, \varphi_{j}\right\rangle$ coefficient. We summarize this result in the next corollary:

Corollary 4. The output impulse response function for the Calvo-plus model depends only on the even-indexed eigenvalue-eigenfunctions $\left(\lambda_{j}, \varphi_{j}\right.$ with $\left.j=2,4, \ldots\right)$, and has zero loadings on the odd-indexed ones, such as the dominant eigenvalue. Thus the first leading term corresponds to the second eigenvalue $\lambda_{2}$. The marginal impulse response is

$$
Y(t)=\sum_{j=2,4,6, \ldots} e^{\lambda_{j} t}\left\langle\bar{p}^{\prime}, \varphi_{j}\right\rangle\left\langle f, \varphi_{j}\right\rangle \text { and } \lambda_{2}=\lim _{t \rightarrow \infty} \frac{\log Y(t)}{t}
$$

where the expressions for the projections are given in equation (22).

Figure 1: Selection effect in the Calvo-plus model
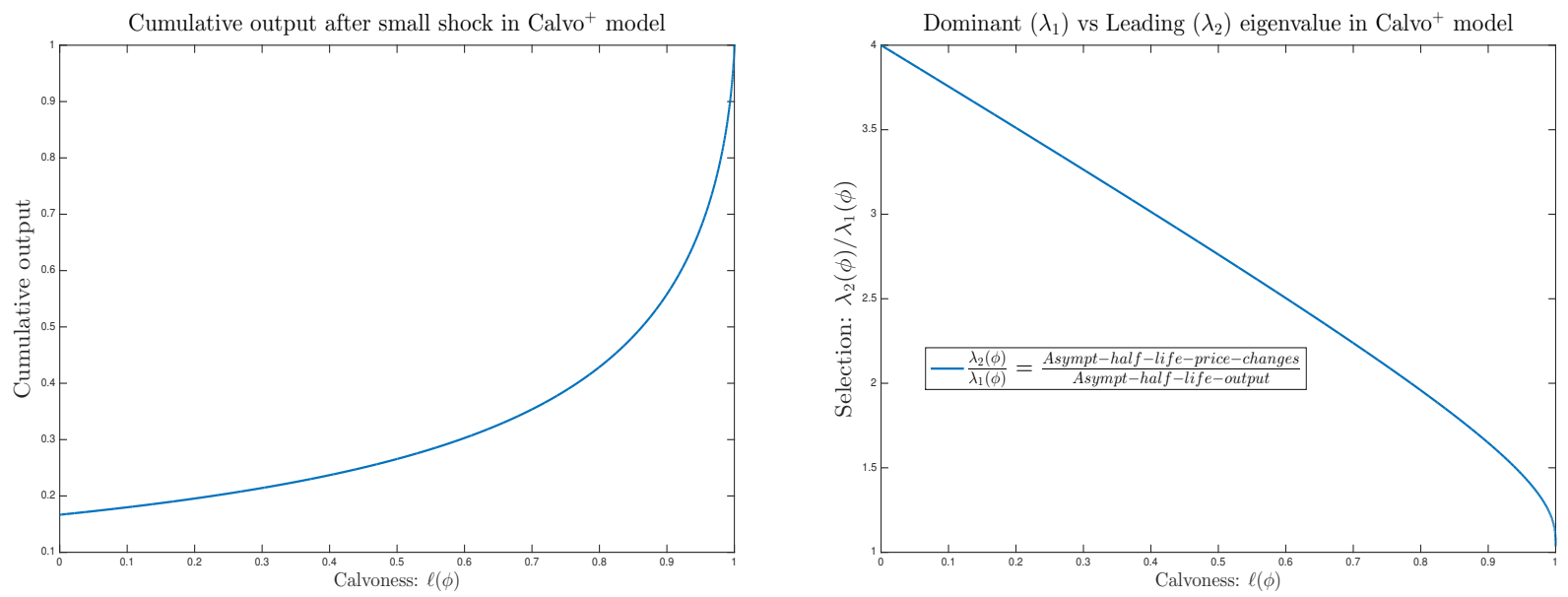

The corollary states that only half of the eigenvalues (those with an even index) show up in the output impulse response function. The largest eigenvalue is $\lambda_{2}$, which we call the "leading" eigenvalue of the output response function. It is interesting to notice that the dominant eigenvalue $\lambda_{1}$ does not appear in the impulse response for output. Notice the difference with the survival function where the only eigenvalues that appear are those with an odd-index. The right panel of Figure 1 plots the ratio between the leading eigenvalue 
for output $\lambda_{2}$ and the dominant eigenvalue $\lambda_{1}$. It is straightforward to see that the ratio is

$\frac{\lambda_{2}}{\lambda_{1}}=\frac{8 \phi+4 \pi^{2}}{8 \phi+\pi^{2}}$ depends only on $\phi$, so that it can be immediately mapped into the "Calvoness" of the problem $\ell(\phi) \in(0,1)$. It appears that the ratio, which can also be interpreted as the ratio between the asymptotic duration of price changes over the asymptotic duration of the output impulse response, is monotonically decreasing in $\ell$, and converges to 1 as $\ell \rightarrow 1$. The economics of this result is that the shape of the impulse response of output depends on the differential impact of the aggregate shock on price increases and price increases. Instead the dominant eigenvalue controls the asymptotic behavior of price changes, both increases and decreases. As $\ell \rightarrow 1$ selection disappears from the model and the two durations coincide. The left panel of the figure uses the particular case of a small monetary shock to illustrate that as $\ell$ increases the cumulated output effect becomes larger due to a muted selection effect.

\subsection{IRF of cross sectional even moments after a monetary shock.}

This section shows that the response of even centered moments to a marginal monetary shocks is zero at all horizons. This applies for instance to the variance of price gaps, or cross-sectional price dispersion, the kurtosis of the price changes, as well as to the survival function. The result is relevant because e.g. the cross-sectional price dispersion is the main measure of inefficiency in models with price stickiness. This result implies that cross-sectional price dispersion is not sensitive to small monetary shocks, so that its fluctuations won't be detectable in estimated impulse responses, but that instead it reacts to changes with the steady state level of inflation, or more generally with the monetary policy regime. An advantage of the analytic approach is to clearly illustrate the forces behind this surprising result.

In many monetary models the presence of price stickiness implies that welfare of the representative consumer depends on the dispersion of prices, or markups (i.e. prices relative to a flexible price benchmark where dispersion is nil). It is therefore of interest to analyze 
how the dispersion of markups behaves following a small monetary shock of size $\delta .{ }^{6}$ Let $M_{k}(t ; \delta) \equiv \mathbb{E}[x(t)-\mathbb{E}(x(t))]^{k}$, for $k=2,4, \ldots$, denote the $k$-th even centered moment of $x$, measured $t$ periods after the monetary shock $\delta$ hits the economy at the steady state. In particular, $M_{2}(t ; \delta)$ will denote the cross sectional variance of markups $t$ periods after the monetary shock. We have the following result:

Proposition 3. Assume the initial condition $\hat{p}$, the signed mass right after the aggregate shock, is anti-symmetric. Then the initial impulse $\hat{p}$ does not have a first-order effect on any even centered moment $M_{k}(t ; \delta)$ with $k=2,4, \ldots$

The proposition implies that a small (marginal) monetary shock does not have a first order impact on the dispersion of markups at all $t>0$ after the monetary shock. It also shows that a zero first-order effect is predicted for all even centered moments of the distribution of markups, such as Kurtosis. The result does not apply to the uneven moments, such as the mean markup (proportional to total output) or the skewness of the distribution, that display a non-zero first order effect following a marginal monetary shock. As mentioned at the beginning of the section this result matters because even moments, such as the dispersion of price gaps, map directly into the efficiency of the economy. In terms of measurement the result implies one should look at the effect of varying the level of inflation on price dispersion as in Alvarez et al. (2019) or Nakamura et al. (2018). Likewise, the hazard rate of price changes should not react to a small monetary shock. This gives a theoretical foundation to the estimation of hazard rates of price changes using unconditional time series evidence. ${ }^{7}$ Alternatively, this prediction can be used to test the model. Finally, we mention that this result also applies to the Kurtosis of price changes because we have argued elsewhere that the steady state kurtosis and frequency of price changes characterize the cumulative IRF to a small monetary shock, see Alvarez, Le Bihan, and Lippi (2016); Alvarez, Lippi, and Oskolkov

\footnotetext{
${ }^{6}$ We are thankful to Nobu Kiyotaki for posing this question to us.

${ }^{7}$ In Alvarez et al. (2019) it is shown theoretically that price dispersion and the average frequency of price changes should not be affected by inflation around zero inflation, but that it should be responsive at higher levels; supporting evidence on both predictions is offered.
} 
(2020).

\subsection{Price-plans and the hump-shaped Output IRF.}

The model with price-plans assumes that upon paying the menu cost the firm can choose two, instead of one price. At any point in time the firm is free to charge either price within the current plan, but changing the plan is costly. The idea was first proposed by Eichenbaum, Jaimovich, and Rebelo (2011) to model the phenomenon of temporary price changes (prices that move from a reference value for a short period of time and then return to it). In Alvarez and Lippi (2019a) we provide an analytic solution to this problem and characterize the determinants of $\bar{x}$, the threshold where a new plan is chosen, as well as the optimal prices within the plan, named $\tilde{x}$ and $-\tilde{x}$. When $x \in[-\bar{x}, 0]$ the firm charges $-\tilde{x}$ and when $x \in(0, \bar{x}]$ it charges $\tilde{x}$. The invariant density of price gaps is still given by equation (20). For a given threshold $\bar{x}$ the value of $\tilde{x}$ is given by: $\tilde{x}=\bar{x}\left[\frac{e^{\sqrt{2 \phi}}-e^{-\sqrt{2 \phi}}-2 \sqrt{2 \phi}}{\sqrt{2 \phi}\left(e^{\sqrt{2 \phi}}+e^{-\sqrt{2 \phi}}-2\right)}\right] \equiv \bar{x} \rho(\phi)>$ 0 where $\phi=\bar{x}^{2} \zeta / \sigma^{2}$. The function $\rho(\phi)$ gives the optimal price within the plan as a function of the adjustment threshold, namely $\tilde{x}=\rho(\phi) \bar{x}$. Simple analysis shows that the images of $\rho(\phi)$ lie in the interval $(0,1 / 3)$, and that $\lim _{\phi \rightarrow 0} \rho(\phi)=1 / 3$.

In this model the output of a firm with gap $x$ is given by $\tilde{f}(x)=-x-\tilde{x}$ if $x \in[-\bar{x}, 0)$ and $\tilde{f}(x)=-x+\tilde{x}$ if $x \in(0, \bar{x}]$. By the linearity of Fourier series we can add to the coefficients of the function $f(x)=-x$, given in equation (16) the ones of the step function: $f_{0}(x)=-\tilde{x}$ if $x \in[-\bar{x}, 0)$ and $f_{0}(x)=\tilde{x}$ if $x \in(0, \bar{x}]$. The function $f_{0}$ has coefficients equal to: $\left\langle f_{0}, \varphi_{j}\right\rangle=-\frac{8 \bar{x}^{3 / 2} \rho(\phi)}{j \pi}$ if $j=2+4 i$ for $i=0,1,2, \ldots$, and $\left\langle f_{0}, \varphi_{j}\right\rangle=0$ otherwise. The expression for the impulse response thus features the additional term

$$
\left\langle\bar{p}^{\prime}, \varphi_{j}\right\rangle\left\langle f_{0}, \varphi_{j}\right\rangle= \begin{cases}0 & \text { if } j \text { is odd or if } j \text { is even and } \frac{j}{2} \text { is even } \\ -4 \rho(\phi)\left[\frac{1+\cosh (\sqrt{2 \phi})}{\cosh (\sqrt{2 \phi})-1}\right]\left[\frac{1}{1+\frac{j^{2} \pi^{2}}{8 \phi}}\right] & \text { if } j \text { is even and } \frac{j}{2} \text { is odd }\end{cases}
$$


Figure 2: Price Plan model

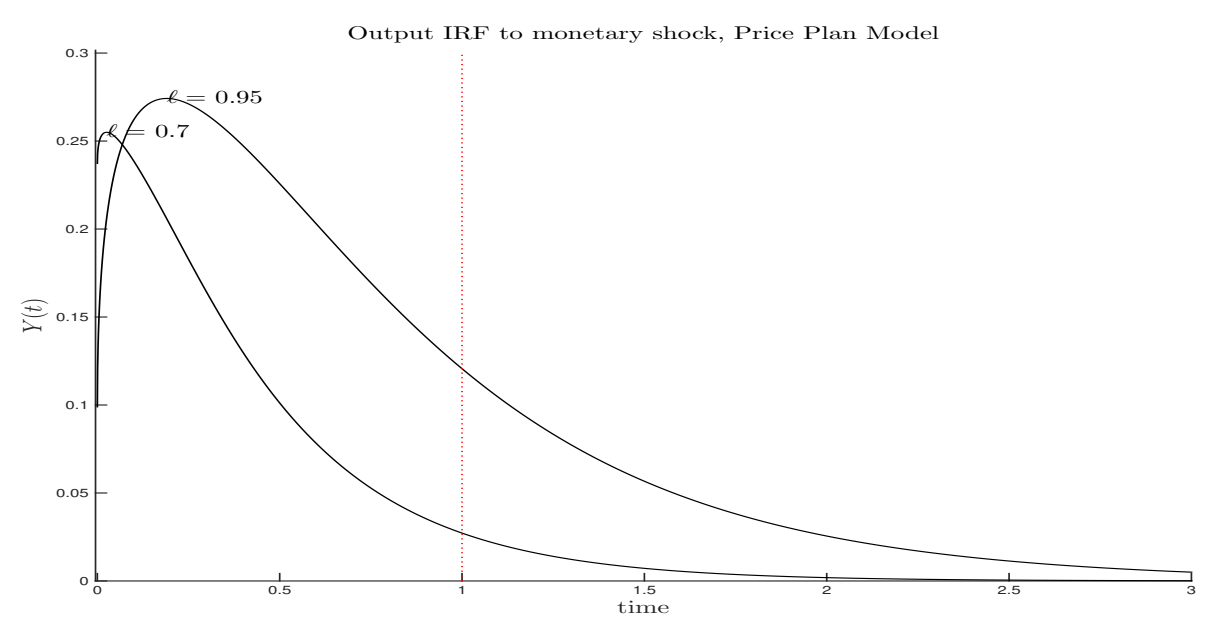

Thus the marginal impulse response is given by:

$$
Y_{\text {Plan }}(t)=Y_{\text {Calvoplus }}(t)+\sum_{j=1}^{\infty}\left\langle\bar{p}^{\prime}, \varphi_{j}\right\rangle\left\langle f_{0}, \varphi_{j}\right\rangle e^{\lambda_{j}(\phi) t}
$$

where the expression for $Y_{\text {Calvoplus }}$ was given in equation (22). While the impulse response is monotone decreasing in the Calvo-plus model, in the price plan model the impulse response can be hump shaped. Indeed, as $\phi$ increases the Calvoness index $\ell \equiv \zeta / N \in(0,1)$ defined before also increases, and the impulse response goes from being decreasing to being humpshaped, as shown in Figure 2 (see Proposition 9 in Alvarez and Lippi (2019b) for a formal proposition).

\subsection{Models with a Generalized Hazard Function}

In a series of influential papers Caballero and Engel (1993, 1999, 2007) proposed models of infrequent (price) adjustment where the firm's behavior is summarized by a generalized hazard function $\xi(x)$, giving the probability of adjustment as a function of the state $x$. The microfoundation for such behavior can be derived from our setup, namely equation (1), or from a setup with random menu costs as in the original papers by Caballero and Engel. The 
generalized hazard function provides a considerable generalization of the Calvo-plus model where the hazard function $\xi$ is constant within the inaction region, as in the case considered in equation (13). Instead, in this more general setup the hazard function $\xi$ depends on the value of the state, as in equation (12). Many authors have employed this set-up, starting with the seminal work by Dotsey, King, and Wolman (1999). ${ }^{8}$

A case considered in the price setting literature is the one where $\xi(x)$ is quadratic and $\bar{x}=\infty$, as in Caballero and Engel (2007) and Berger and Vavra (2018). For this case all the eigenvalues and eigenfunctions are known analytically, as shown below. Another tractable example is the one where the hazard function is the absolute value, $\xi(x)=\kappa|x|$, a case that is strongly supported by the estimates of Figure 8 and 9 in Eichenbaum, Jaimovich, and Rebelo (2011). The eigenvalues and eigenfunctions for this case are known to be the Airy functions and its zeroes. More broadly, we note that there is a wealth of analytical approximations used in quantum mechanics to characterize both eigenvalues and eigenfunctions for this type of equations, including perturbation methods which expand $\xi$ around a known solution, expansion methods around small values of $\sigma^{2}$, and approximation methods that replace $\xi$ by a piecewise constant functions, as discussed in Appendix B.

The quadratic generalized hazard. Consider the quadratic generalized hazard for the symmetric unbounded case i.e. where $\xi(x)=\xi_{0}+\frac{1}{2} \xi_{2} x^{2}$ where $\xi_{0}$ and $\xi_{2}$ are nonnegative parameters characterizing the hazard function. Let $\left\{\lambda_{j}, \varphi_{j}\right\}$ be the eigenvalues and eigenfunctions that solve equation (12) for this $\xi(x)$. After a change of variables this is exactly the equation for the eigenvalues (energy levels) and eigenfunctions (eigenstates) of the quantum harmonic oscillator, a well known problem in physics whose solution we exploit for the next result:

\footnotetext{
${ }^{8}$ In Alvarez, Lippi, and Oskolkov (2020) we characterize price setting in the case with no drift and with a symmetric instantaneous profit function. We have shown that any symmetric increasing function $\xi(x)$ can be rationalized by some distribution of menu cost, and also shown that $\xi$ itself is uniquely identified by the steady state distribution of price changes. Moreover, we have shown that the cumulative impulse response of output for an economy where price setting is given by any such generalized hazard function equals the Kurtosis of the price changes dividend by six times the frequency of price changes.
} 
Proposition 4. The eigenvalues and eigenfunctions $\left\{\lambda_{j}, \varphi_{j}\right\}$ that solve equation (12) with $\xi(x)=\xi_{0}+\frac{1}{2} \xi_{2} x^{2}$ and $\bar{x}=-\underline{x}=\infty$ and $\mu=0$ are given by:

$$
\begin{aligned}
\lambda_{j} & =-\left[\sigma \sqrt{\xi_{2}}\left(j-\frac{1}{2}\right)+\xi_{0}\right] \text { for all } j=1,2, \ldots \\
\varphi_{j}(x) & =\left(2^{j-1}(j-1) ! \sqrt{\pi}\right)^{-\frac{1}{2}} \sqrt{\eta} e^{-\frac{\eta^{2} x^{2}}{2}} H_{j-1}(\eta x) \text { for all } x
\end{aligned}
$$

where $\eta \equiv\left(\xi_{2} / \sigma^{2}\right)^{1 / 4}$ and $H_{j}(\cdot)$ is the (physicist's) Hermite polynomial of degree $j$, given by $H_{j}(x)=(-1)^{j} e^{x^{2}} \frac{d^{j}}{d x^{j}} e^{-x^{2}}$.

It is immediate to see that, as was the case for the Calvo-plus model, the constant $\xi_{0}$ simply adds to each of the eigenvalues obtained for $\xi_{0}=0$ case, and does not affect the eigenfunctions.

Figure 3: Output response and hazard rates in different models (with $N=1$ )

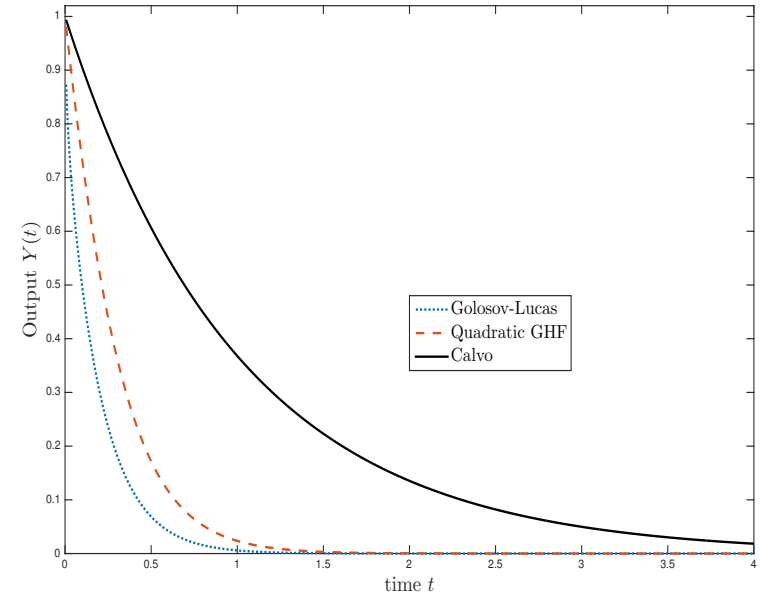

Output impulse response $Y(t)$

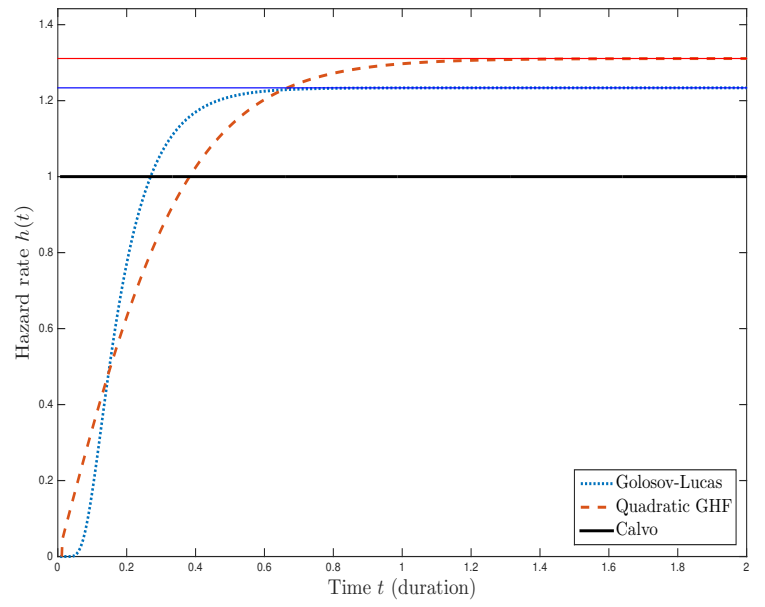

Hazard function $H(t)$

We use the proposition to compare the impulse response function generated by three different models: the Golosov and Lucas model described in equation (18), a model with quadratic hazard (where $\xi_{0}=0$ and $\xi_{2}=1$ ), and a Calvo model (with constant hazard rate $N)$. All models feature the same number of price adjustments per unit of time, so that we normalize $N=1$ without loss of generality. The left panel of Figure 3 plots the impulse 
response produced by each model. It is apparent that the output response in the quadratic hazard model (dashed line) is close to the one by Golosov and Lucas (dotted line), and much smaller than the one in Calvo (solid line).

Following the logic used in Corollary 3 we analytically compute the survival function and the hazard rate of adjustment as a function of durations, which are reported in the right panel of the figure (see Appendix B for the analytic solution). We proved in Corollary 4 that the dominant eigenvalue is irrelevant for the characterization of the output response, since its associated eigenfunction has a zero projection with respect to the (antisymmetric) output function. However, one might conjecture that the dominant eigenvalue might still contain useful information on the model dynamics. The right panel of the figure plots the dominant eigenvalue, which corresponds to the asymptotic value of the hazard function marked by the thin horizontal lines (as established in Corollary 3). This example shows that the ranking of the dominant eigenvalue is not matched by the ranking of the persistence of the monetary non-neutrality. The quadratic hazard model features the largest eigenvalue, in spite of the fact that the propagation in that model is in between Calvo and Golosov-Lucas.

The analytic results reveal the robustness of the patterns portrayed in the figure. The three dominant eigenvalues for these models are given by the expressions that feature only one parameter, namely the frequency of price adjustment $N .{ }^{9}$ Thus, for models with the same $N$, there is no other parameter affecting the magnitude of the dominant eigenvalue, so that the ranking between the dominant eigenvalues of these three models is always the one reported in the right panel of the figure.

\subsection{More applications}

We briefly mention other applications that are of interest and can be solved using Theorem 1. One application studies how changes to the volatility of shocks affect the propagation of

\footnotetext{
${ }^{9}$ The dominant eigenvalue, $\lambda_{1}$, in Calvo is $\lambda_{\text {Calvo }}=-N$, in the quadratic hazard model it is $\lambda_{Q u a d}=$ $-\sqrt{\frac{8}{\pi}} \Gamma\left(\frac{5}{4}\right)^{2} N \approx-1.31 N$, while in Golosov-Lucas it is: $\lambda_{G L}=-\pi^{2} / 8 N \approx-1.23 N$.
} 
monetary innovations. The issue matters to e.g. the effectiveness of monetary policy in recessions vs boom, when the state of the economy is assumed to feature, respectively, high vs low volatility of shocks as in Vavra (2014). Our method provides a sharp analytic answer to this question (see Appendix $\mathrm{C}$ for details).

We also mention a model not included by setup above, where the eigenvalues and eigenfunctions can be explicitly computed is the one of firm facing a multiproduct price-setting problem, as in Midrigan (2011) for two problems or the general case of $n$ products considered in Alvarez and Lippi (2014). This case is analyzed in Appendix D, where the $n$-dimensional problem is reduced to a two dimensional case.

\section{Scope: local insensitivity to asymmetries}

In this section present a result that is useful to frame the scope of Theorem 1 . We show that the output's impulse response to a marginal monetary shock is locally insensitive to two forms of asymmetries. The first one concerns deviations from the zero drift, or zero inflation, assumption. The second concerns the deviation from the symmetry of the firm's objective function. The insensitivity applies to the impulse response of any anti-symmetric function $f$, not just the one for output, following a marginal monetary shock. This gives the precise sense in which our result for a symmetric $s S$ problem extends to a range of small inflation rates.

We let $\mu$ be the steady state inflation. We also let $a$ be a coefficient that measures the degree to which the firms's period return function is asymmetric. In particular, we let $R(x, a)$ be the return function which satisfies

$$
R(x, a)=R(-x,-a) \text { for all } x, a
$$

Note that $a=0$ implies that the return function is symmetric. For $a \neq 0$, the function can be interpreted as the sum of a symmetric and anti-symmetric function. An example of this 
is $R(x, a)=B x^{2}+a x^{3}$ which can represent a third order expansion of the original profit function around the static maximizing choice $x=0$. In this case $a$ is $1 / 6$ times the third derivative of the original profit function at $x=0$. When either $a \neq 0$ or $\mu \neq 0$ the thresholds of the optimal Ss rule will not be equidistant from the optimal return point $x^{*}$.

The next proposition computes two derivatives of the output's IRF to a small monetary shock at any horizon $t$ and shows that they are both zero. One is the derivative with respect to steady state inflation, and the other one is the derivative with respect to the degree of asymmetry of the objective function. For this proposition we need to consider the general case with re-injection, since we don't have the required conditions of Proposition 1 that ensure that $H=G$. Also note that the steady state distribution $\bar{p}$ will be, in general, also a function of the drift and the degree of asymmetry $(\mu, a)$, and hence we include them as arguments of the initial impulse $\hat{p}(x ; \delta, \mu, a)$, as well as of the function $H$ itself, since this function depends on the process for $x$, and in particular the value of its drift, optimal return point $x^{*}$, and thresholds $\underline{x}, \bar{x}$. We also explicitly include the shock $\delta$ as an argument of $\hat{p}$, which will clarify the arguments below. The initial condition for a monetary shock are thus $\hat{p}(x ; \delta, \mu, a) \equiv \bar{p}(x+\delta ; \mu, a)-\bar{p}(x ; \mu, a)$. Thus the marginal impulse response is:

$$
\left.Y(t ; f, \mu, a) \equiv \frac{\partial}{\partial \delta} H(t ; f, \hat{p}(\cdot, \delta, \mu, a), \mu, a)\right|_{\delta=0} \text { for all } t \geq 0
$$

The next proposition states that impulse response $Y(t ; f, \mu, a)$ at any horizon $t$ is approximately the same as our benchmark case with zero inflation and with symmetric return function. Formally we have:

Proposition 5. Consider, for simplicity, the Calvo-plus model. Let $\mu$ be the drift of the state (the inflation rate), and $a$ the index of asymmetry in the return function $R(x, a)$. Then:

$$
\left.\frac{\partial}{\partial \mu} Y(t ; f, \mu, a)\right|_{\mu=0, a=0}=0 \text { and }\left.\frac{\partial}{\partial a} Y(t ; f, \mu, a)\right|_{\mu=0, a=0}=0 \quad \text { for all } t \geq 0
$$

for any anti-symmetric function $f$. 
See Appendix A for the proof. This result establishes the insensitivity of the marginal impulse response function with respect to small amounts of inflation and asymmetries in the objective function. Notice that the proof holds for any function of interest $f$ that is antisymmetric. So this holds true for the output impulse response, given by $f(x)=-x$, as well as for other anti-symmetric functions.

\section{Conclusion and Future Work}

We used an eigenfunction-eigenvalue decomposition technique to analytically characterize the impulse response function for a large class of sticky price models. We illustrated the usefulness of this method with several applications. The main results were derived for problems "without reinjection", an assumption that simplifies the analysis and allows us to consider moderate degrees of asymmetry in the problem of interest.

Open questions for future research involve exploring setups featuring asymmetries and large drift, as well as problems with non-negligible strategic complementarities. ${ }^{10}$ For instance, asymmetries are important in inventory models, or problems where drift is important, as in models of capital relocation with frictions. Strategic complementarities can also be important depending on the general equilibrium setup faced by firms. The analysis of problems featuring both asymmetries and drift requires working out the dynamics of problems with reinjection, for which we already derived some result in this paper (see Appendix E), but more is needed. Incorporating strategic complementarities requires extending the result to endogenous moving boundary problems. In ongoing work, Alvarez, Lippi, and Souganidis (2021) we show that the use of Mean Field Games methods can be a promising avenue to analyze such problems analytically. Interestingly, the solutions derived here are essential, since the problem with complementarities will be solved using a perturbation around the problem with no complementarities studied in this paper.

\footnotetext{
${ }^{10}$ Our characterization uses a general equilibrium environment which implies that there are no first order strategic complementarities in the firm's optimal decision rules.
} 


\section{References}

Alexandrov, Andrey. 2020. "The Effects of Trend Inflation on Aggregate Dynamics and Monetary Stabilization." Mimeo, University of Mannheim.

Alvarez, Fernando E., Martin Beraja, Martin Gonzalez-Rozada, and Pablo Andres Neumeyer. 2019. "From Hyperinflation to Stable Prices: Argentina's Evidence on Menu Cost Models." The Quarterly Journal of Economics 134 (1):451-505.

Alvarez, Fernando E., Herve Le Bihan, and Francesco Lippi. 2016. "The real effects of monetary shocks in sticky price models: a sufficient statistic approach." The American Economic Review 106 (10):2817-2851.

Alvarez, Fernando E. and Francesco Lippi. 2014. "Price setting with menu costs for multi product firms." Econometrica 82 (1):89-135.

—. 2019a. "Temporary Price Changes, Inflation Regimes and the Propagation of Monetary Shocks." American Economic Journal: Macroeconomics in press.

_ 2019b. "“The Analytic Theory of a Monetary Shock"." Working Paper 19, EIEF.

Alvarez, Fernando E., Francesco Lippi, and Aleksei Oskolkov. 2020. "The Macroeconomics of Sticky Prices with Generalized Hazard Functions." Working paper, EIEF.

Alvarez, Fernando E., Francesco Lippi, and Panagiotis Souganidis. 2021. "Price Setting with Strategic Complementarities as Mean Field Games." Mimeo, University of Chicago.

Baley, Isaac and Andres Blanco. forthcoming. "Aggregate Dynamics in Lumpy Economies." Econometrica .

Berezinn, F.A. and M. Shubin. 1991. The Schrodinger Equation. Springer Science+Business Media Dordrecht - Mathematics and Its Applications (Soviet Series).

Berger, David and Joseph Vavra. 2018. "Dynamics of the U.S. price distribution." European Economic Review 103:60 - 82.

Bhattarai, Saroj and Raphael Schoenle. 2014. "Multiproduct Firms and Price-Setting: Theory and Evidence from U.S. Producer Prices." Journal of Monetary Economics 66:178-192.

Borovicka, Jaroslav, Lars Peter Hansen, and Jos A. Scheinkman. 2014. "Shock elasticities and impulse responses." Mathematics and Financial Economics 8 (4):333-354.

Caballero, Ricardo J. and Eduardo M. R. A. Engel. 1999. "Explaining Investment Dynamics in U.S. Manufacturing: A Generalized (S,s) Approach." Econometrica 67 (4):783-826.

Caballero, Ricardo J. and Eduardo M.R.A. Engel. 1993. "Heterogeneity and output fluctuations in a dynamic menu-cost economy." The Review of Economic Studies 60 (1):95.

- 2007. "Price stickiness in Ss models: New interpretations of old results." Journal of Monetary Economics 54 (Supplement):100-121. 
Calvo, Guillermo A. 1983. "Staggered prices in a utility-maximizing framework." Journal of Monetary Economics 12 (3):383-398.

Costain, James and Anton Nakov. 2011. "Price Adjustments in a General Model of StateDependent Pricing." Journal of Money, Credit and Banking 43:385-406.

Dotsey, Michael, Robert G. King, and Alexander L. Wolman. 1999. "State-Dependent Pricing And The General Equilibrium Dynamics Of Money And Output." The Quarterly Journal of Economics 114 (2):655-690.

Eichenbaum, Martin, Nir Jaimovich, and Sergio Rebelo. 2011. "Reference Prices, Costs, and Nominal Rigidities." American Economic Review 101 (1):234-62.

Fernandez, Francisco M. 2001. Introduction to Perturbation Theory in Quantum Mechanics. CRC Press, 1st Edition.

Gabaix, Xavier, Jean-Michel Lasry, Pierre-Louis Lions, and Benjamin Moll. 2016. "The Dynamics of Inequality." Econometrica 84 (6):2071-2111.

Gertler, Mark and John Leahy. 2008. "A Phillips Curve with an Ss Foundation." Journal of Political Economy 116 (3):533-572.

Golosov, Mikhail and Robert E. Jr. Lucas. 2007. "Menu Costs and Phillips Curves." Journal of Political Economy 115:171-199.

Hansen, Lars Peter and José A. Scheinkman. 2009. "Long-Term Risk: An Operator Approach." Econometrica 77 (1):177-234.

Midrigan, Virgiliu. 2011. "Menu Costs, Multi-Product Firms, and Aggregate Fluctuations." Econometrica, 79 (4):1139-1180.

Nakamura, Emi and Jon Steinsson. 2010. "Monetary Non-Neutrality in a Multisector Menu Cost Model." The Quarterly Journal of Economics 125 (3):961-1013.

Nakamura, Emi, Jon Steinsson, Patrick Sun, and Daniel Villar. 2018. "The Elusive Costs of Inflation: Price Dispersion during the U.S. Great Inflation*." The Quarterly Journal of Economics 133 (4):1933-1980.

Taylor, John B. 1980. "Aggregate Dynamics and Staggered Contracts." Journal of Political Economy 88 (1):1-23.

Vavra, Joseph. 2014. "Inflation Dynamics and Time-Varying Volatility: New Evidence and an Ss Interpretation." The Quarterly Journal of Economics 129 (1):215-58.

Woodford, Michael. 2009. "Information-Constrained State-Dependent Pricing." Journal of Monetary Economics 56:s100-s124.

Zettl, Anton. 2010. Sturm-Liouville Theory. American Mathematical Society. 